\title{
SEGMENTED BURST SWITCHING: ENHANCEMENT OF OPTICAL BURST SWITCHING TO DECREASE LOSS RATE AND SUPPORT QUALITY OF SERVICE
}

\author{
Abdelilah Maach, Gregor von Bochmann \\ Department of Computer Science, School of Information Technology \& Engineering, University \\ of Ottawa, Canada \\ \{amaach, bochmann $\}$ asite.uottawa.ca
}

\begin{abstract}
With the growing demand of bandwidth and the development being made in the optical components technology, the $\mathbb{P}$ over DWDM using optical burst switching seems to be the best solution to take advantage of the huge capacity of the fiber and accommodate high traffic of information. In this architecture the optical network is seen as an optical cloud with intelligent edges capable to interpret the $\mathbb{P}$ address and store the information in electronic domains as well as checking and correcting errors. Optical burst switching [1,3] achieves better bandwidth exploitation because all the fiber wavelengths are shared among the bursts without resource pre-allocation and the whole wavelength capacity can be used by a burst. However with higher load, contention increases, and hence the number of dropped burst increases leading to a big lost of performance. Several methods can be used to lower the burst-dropping probability such as wavelength conversion [5] and the use of buffers [6], but these solutions are still not there due to the high cost and the immaturity of technology. In this paper we will propose another method where the burst is segmented into several parts of equal length and in case of contention only the parts at the beginning, causing the conflict, will be discarded. We will analyze and compare this method with other practical methods: delayed burst, deflection routing. The segments of burst can be used to carry different class of service since the dropping probability of each segment depends on its position in the burst. The parts at the end have the smallest probability to be dropped. We prove trough analysis and simulation that the segmented burst improves the performance and is more suitable for traffic with several class of service.
\end{abstract}


Key words: Optical network, optical burst switching, contention resolution, segmented burst switching

\section{INTRODUCTION}

Wavelength division multiplexing WDM consists of the use of several light paths in the same fiber. This increases tremendously the capacity of the fiber, which makes it a good solution to face the increasing demand of bandwidth. At the beginning the network consisted of electronic switch connected by optical fibers. At each intermediate node, the data has to be converted to the electronic domain and stored temporarily while the header is being processed. Unfortunately the electronic processing and conversions limits the speed of the network, and doesn't exploit all the advantages of WDM. Furthermore the network cost is very high due to the components used to perform opto-electronic conversion and storage. The trend now is to keep the information as long as possible in the optical domain, which can overcome the limitations imposed by the electronic processing and optoelectronic conversion as well as the buffering problems while providing high speed, high transparency and decreases the network cost. The trend in photonic network is to carry $\mathbb{P}$ traffic directly over the DWDM.

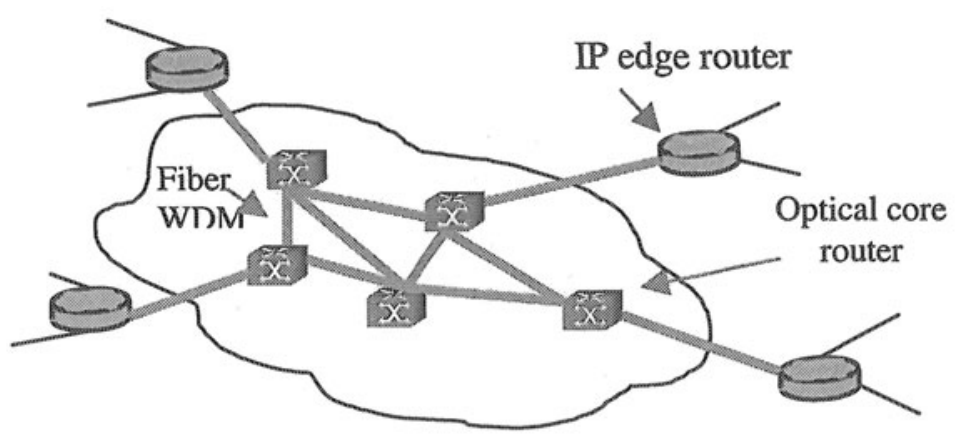

Figure1. An optical network with $\mathbb{I P}$ edge routers and optical switch inside the network

Figure 1 shows an IP network operating over an optical backbone. So far all-optical network is not available, but the big progress in optical technology, especially in optical components such as add/drop and OXC, 
makes it possible to have what is called almost-all optical network [7]; in this kind of network only a small part of the traffic is converted to the electronic domain whereas the big part remains in the optical domain. Several techniques and methods have been proposed to carry information using this kind of networks; among them we find re-configurable [8,9] networks, which consist of a logical topology built over the physical one by the assignment and allocation of wavelength, and reconfiguration is performed dynamically to meet the traffic load. In general, the goal of reconfiguration is to minimize the average number of hops to reach destination. Another method consists of keeping information in optical form and performing the burst switching instead of packet switching. Optical burst switching remains the best method and promises to provide a very reliable network. The basic difference between the conventional network and the optical switcher is the fact that in the former all the switches have the capacity to store information and participate to manage and monitor the network. With this distributed architecture the network can face all the situations and regulate the load of network by using explicit methods to control the data flow and regulate the load. However, in the optical burst switching all the intelligence is pushed to the edges which are at the same time the buffer and the processor of the network, whereas the intermediate node are used to forward messages according to their destination. But at these node conflict $[10,1]$ can occur when multiple bursts are received that need to egress on the same port and wavelength at the same time. In case of contention the simplest solution is to discard the second bursts and keep only the first one. This can degrade the network performance and lead to a big waste of bandwidth. In this paper we propose a solution that reduces the network latency and enhances bandwidth. This solution consists of subdividing the burst into several parts to avoid the dropping of the whole burst; only the contented segments will be discarded. Besides by its small length, the segment can fit easily to fill the blank between two bursts, and hence this will give more fluidity to the traffic. The segments can be used also to carry different class of service.

We will compare segmented optical burst switching to two other methods:

1. Using node with limited buffer: in this case every node in the network will have a small buffer to delay the contented burst.

2. Deflection: in this case, the node instead of dropping the burst will use an alternative path; this is possible by sending the burst on another free output.

In this work we will analyze the network performance with different load, when using different methods to decrease the blocking probability. Also we prove through simulation that optical segmented burst switching 
SBS will give always good result, even better, the segment at the end of a burst is often saved. The rest of this paper is organized as follows. Section 2 presents the optical burst switching concept. Section 3 discusses contention and its impact on the performance. Section 4 presents optical segmented burst switching and its benefits. Section 5 presents some simulation result and the variation of performance with the network load and the switch output number. Section 6 concludes this paper.

\section{OPTICAL BURST SWITCHING TECHNIQUES}

In an optical network using optical burst switching technique, the edge nodes are able to store and process $\mathbb{I P}$ packet whereas the intermediate nodes will perform forwarding according to the egress destination. In this architecture the incoming packets are buffered, in the source edge routers, to form bursts. Bursts are collected according to their destination and class of service. Then, a controller packet is sent over a specific optical wavelength channel to announce an upcoming burst. The controller packet, called also an optical burst header $\mathrm{OBH}$, is then followed by a burst of data without waiting for any confirmation. The $\mathrm{OBH}$ is converted to electrical domain at every node to be interpreted and transformed according to the routing decision taken at each switching node, and pertinent information is extracted such as the wavelength used by the following data burst, the time it is expected to arrive, the length of the burst and the label, which determines the destination. This information will be used by the switch to schedule and setup the transition circuit for the coming data burst. There are several approaches to OBS, nonetheless the difference is related to the timing issues concerned with synchronization between the data bursts and their headers. Those techniques fall into two categories:

1. OBS with offset: in this category, the data burst is delayed at the source $[11,12]$ and sent after a short delay as shown in figure 2a. This offset must be long enough to cover the processing time at all intermediate nodes. This assumes that the source knows the number of hops needed to reach the destination and the processing time at each node. The advantage of this method is that no delay lines are needed at the intermediate node. Furthermore, the offset can be used to define several classes of services [13]; indeed an extra offset is added to the burst to give it higher priority, the longer the offset is the higher its priority.

2. OBS without offset: in this category the data burst is sent at the same time with the $\mathrm{OBH}$ as shown in figure $2 \mathrm{~b}$ and delayed at the switch by a fiber delay line (FDL). The delayer in this case is different from the 
buffer since we don't need to store the burst. This method has the advantage to free the edge from the burden of calculating the offset.

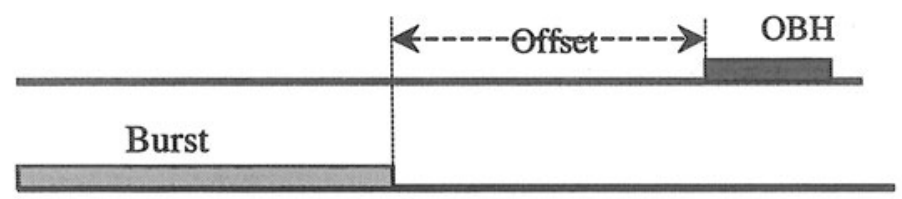

Figure $2 a$. The burst will be delayed at the source

$\mathrm{OBH}$

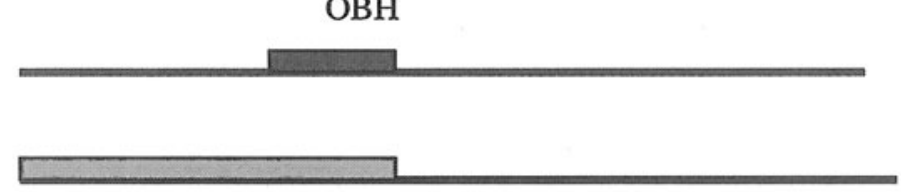

Figure $2 b$. The burst will be delayed at the source

Normally the $\mathrm{OBH}$ is processed electronically; it may be in the future processed optically. But whether in optical or electronic form, the most important feature is the switching speed since the switching time is considered as a waste of bandwidth.

The routing principle [14] of OBS is similar to the one used by Multi Protocol Label Switching (MPLS) in the sense that both, OBS and MPLS, use a label to forward the data, the label edge routers (LERs) are the edge electronic routers and the label switching routers (LSRs) are replaced by optical cross-connects (OXCs). An OXC is a path switching element that establishes routed paths for optical channels by locally connecting an optical channel from an input port (fiber) to an output port (fiber) on the switch element. Thereby OBS can take advantage and exploits recent advances in the MPLS control plane in term of routing protocols, traffic management and quality of services. Nevertheless there are structural differences between LSRs and OXCs. Is that with the former the forwarding information is carried explicitly as part of the labels appended to data packets, while with the later the switching information is sent separately within another wavelength. Besides OXCs don't perform packet level processing in the data plane, while the LSRs are datagram devices which may perform certain packet level operations in the data plane. These differences may incur some enhancement to adapt MPLS to the new environment especially to deal with the problems of quality of services and traffic engineering.

Another advantage of OBS is that it may be adapted easily to support the multicasting $[15,16]$, indeed the multicasting tree can be constructed at the 
WDM layer and uses light splitting at nodes which is more efficient than the copying technique used by IP multicasting in the electronic domain.

The Optical burst switching combines the intelligence and the capacity of processing and buffering of the electronic edges with the capacity of optical forwarding of the core. Thereby the network can face the increasing traffic and accommodate a different kind of information.

Basically OBS is designed to avoid the long end-to-end set-up times of conventional virtual circuit configuration with no need for memory at intermediate node. However, the big problem is contention, which may occurs when one or more bursts arrive at the same time and try to leave through the same output, using the same wavelength, of course. The way this contention is resolved can affect tremendously the performance of the network. Indeed when the packet-loss ratio increases the efficiency decreases as well as the delivery rate and hence the throughput. In other words the contention make the delivery not guaranty and not deterministic.

\section{DEALING WITH CONTENTION}

Contention is inherent to OBS technique, which basically assumes that the network is buffer-less. This feature makes it quite different from a packet switching network. Indeed in the electronic switch, contention is resolved by the buffering mechanism, which simply keeps the messages in memory and postpones their forwarding until the contented output gets free. But this technique requires enough memory to face the heavy traffic, on the other hand, network management becomes more complicated in order to keep the load balanced and achieve fairness among the waiting packets according to the quality of services and the priority. This cannot avoid completely the packet dropping, in some situations when the memory of the switch is overload, the incoming packet may be dropped.

The simplest schemes to resolve contention in the OBS are the wavelength conversion, the use of buffers and deflection routing:

- Burst buffering: optical memories are not available so to store information, conversion to electronic can be used in a hybrid architecture, this need a fast electronic component to perform this conversion as fast as possible. But this solution is not suitable for OBS, which aims conceptually to keep the data burst in optical domain; also this solution increases the network cost. Another solution consists of using a fiber delay line (FDL), which is quite different from the electronic RAM, nevertheless it could be used to delay the incoming burst. Several solutions have been proposed to introduce efficiently FDL in the switch 
that allow different burst length and share optimally these FDL between all the burst. However so far the FDL remains very cumbersome and add more complexity to the optical switch. Besides used with OBS, FDL may introduce additional latency and limit the flexibility of OBS by limiting the length of data burst. However limited FDL can improve the performance. The basic idea is that some burst can be saved if it could be delayed for a short time. This situation may occur when one burst arrives at the end of another and contend for the same output. The second burst will be switched to the FDL just to avoid the contention. Simulation proves that, depending on the capacity of FDL used, the dropping burst ratio can be reduced.

- Wavelength conversion [17]: consists of the use of additional component that can shift the wavelengths of incoming optical signals. This is an elegant solution to improve the performance without need to additional buffers. Indeed the architecture with converters uses, without limitation, all the resources available in term of bandwidth. It is also most suitable for the OBS since it does not introduce an additional latency. However the technology is still not there, making it too expensive and difficult to implement this technique. It is possible to reduce the network cost by using limited wavelength conversion.

- Deflection routing [18]: the use of alternative path is also a good solution to avoid the systematic burst drop. This is possible by taking an alternative path, which is not necessarily the best one; the routing table must provide more than one output for each destination. This technique tends to balance the network load and uses the network fiber as buffer. However used with OBS, this solution may introduce an additional latency and increases network traffic. Also as the number of hops is not known, the source can find it difficult to compute the offset between the $\mathrm{OBH}$ and data burst, this difficulty can be overcome by using FDL to give an additional offset to the burst in case of deflection. It gives good result if the load is not very high and the number of output is not too small.

In the following we will propose another approach to deal with the contention, and afterward we will compare the method we propose to OBS with limited FDL and OBS with deflection. 


\section{SEGMENTED OPTICAL BURST SWITCHING}

The contention depends on the physical topology and resources available such as the number of wavelength and the network connectivity. But also the contention depends on the burst length and the traffic load. The longer the average burst length is, the higher is the blocking probability. In OBS the edge router assembles the incoming into a unique burst, once sent the burst will reach the destination or all the information in the burst will be lost in case of conflict. On the other hand, if the average length of burst is short, the switch will spend more time to set-up circuit than to carry information, that lead to a big waste of bandwidth. To take advantage of both short and long length we propose another variant of OBS, which use segmented burst instead of the burst. With the segmented burst the ingress routed will group incoming packets into a list of segments with constant length (one to five $\mathbb{P}$ packet in a segment). All the list of segments will be sent with the same $\mathrm{OBH}$ and with a short time between two segments. In case of contention instead of dropping all the burst, only the contented segments, belonging to the second burst, will be discarded whereas the rest of segments can continue their way as shown in figure 3. The information carried by the $\mathrm{OBH}$ are the same, we need just to indicate the number of segments in a burst instead of the length of burst, and this information may change at intermediate nodes whenever some of segments are dropped.

Figure 3 show contention between two segmented burst and how a part of burst may be saved instead of dropping all the burst.

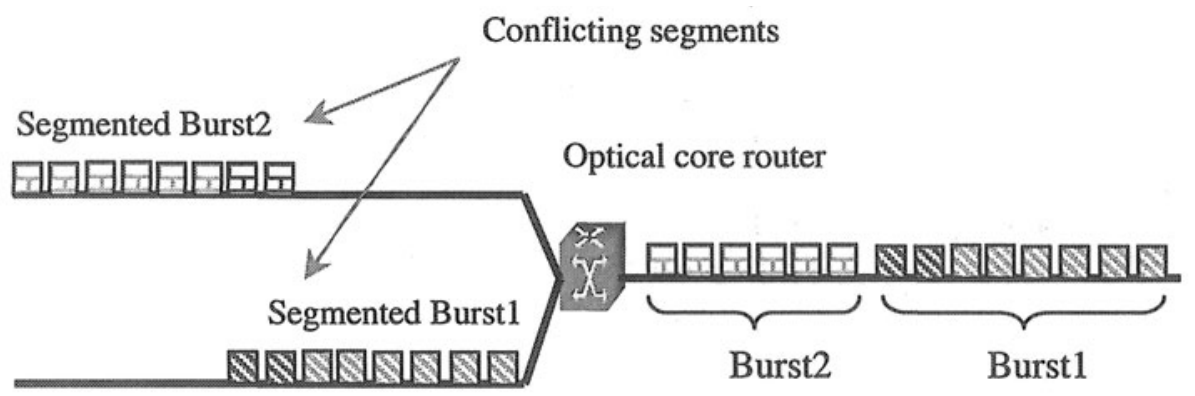

Figure 3 Contention in optical segmented burst switching

Segmented burst adds more flexibility to OBS, and improves the throughput of network since it avoids retransmission and saves some parts of the dropped burst. Unlike buffering and deflection, Segmented burst does not introduce neither additional latency to burst nor additional load to the network. In buffer-less optical network, the scheduler in OBS is constraint to 
respect the arrival time of the bursts; this can lead to unused slots of time between successive bursts.

Example: The scheduler receives three optical burst headers with the following information:

- Burst 1 will arrive at 0.4 and end at 0.6

- Burst 2 will arrive at 0.5 and end at 0.7

- Burst 3 will arrive at 0.7 and end at 0.9

All the bursts want to eave through the same output port. If the node cannot delay the second burst, it will be dropped. Consequently burst 1 and burst 3 can leave without problem, however between 0.6 and 0.7 the egress will be free and unused, which is considered a wasted resource.

The SOBS overcomes this problem; it will take some segments from the burst 2 to fill the blank.

Using the segment granularity, the traffic becomes more fluid, so the output capacity can be used completely, the efficiency will be limited only by the switching time.

Another concern is that the quality of service provided by OBS as there is no criterion to take in consideration when conflict occurs, some approaches have been already proposed in [13] that give better quality of service to traffic which need high priority by assigning additional offset to the burst. However, in that scheme contention will always remain among bursts belonging to the same classes. Besides the OBS flexibility is lost as the burst length may be limited by the offset of other classes.

With segmented bursts, the segment at the end of a burst has more probability to be saved than the others, as we drop the segment at the beginning of the second burst. The higher the position in the list of segments the smaller is the probability to be discarded. This important feature can be used to provide different quality of service, indeed at the ingress edge incoming information are sorted according to their class of services ( CoS ) and destination. Information with high priority will occupy the last segments in the burst.

Table1 gives a comparison between the different forwarding methods.

Table 1. A comparison between the 5 optical switching methods

\begin{tabular}{lllll}
\hline Method & $\begin{array}{l}\text { Additional } \\
\text { Latency }\end{array}$ & $\begin{array}{l}\text { Additonal } \\
\text { Hlardware }\end{array}$ & Support QOS & Complexity \\
\hline Basic OBS & No & No & Yes & No \\
$\begin{array}{l}\text { OBS with } \\
\text { buffer }\end{array}$ & Yes & Yes & No & No \\
$\begin{array}{l}\text { OBS with } \\
\text { deflection }\end{array}$ & Yes & No & No & No \\
$\begin{array}{l}\text { OBS with } \\
\text { conversion }\end{array}$ & No & Yes & No & Yes \\
OBS segment & No & No & Yes & No \\
\hline
\end{tabular}




\section{PERFORMANCE EVALUATION}

As mentioned before, contention is a serious problem, which can affect tremendously the network performance when the OBS is used to route information.

In this section, we will focus on the comparison and performance evaluation of efficient OBS protocols that can reduce burst dropping probability. These techniques are OBS with limited FDL, OBS with deflection and segmented burst. We will also study how segmented bursts are suitable for carrying different classes of traffic, especially the rate of saved segment among those, which would be dropped by basic OBS.

The OBS techniques are traffic load and network topology sensitive, thereby the simulation will take in consideration these parameters; The traffic load will be measured in term of arrival burst frequency and burst length. The network topology will be represented by the number of outputs and the number of fiber by output.

We consider a system with a single switch with high speed switching capacity, and assume that data burst are arriving over each link with a Poisson rate $\lambda$.

We assume also that this switch does not support wavelength conversion, therefore there is no interaction between the wavelengths, and the simulated switch will contain only one wavelength.

The system generates a list of bursts. Each burst is characterized by an arrival time, a length and the destination, which is one of the outputs;

- The arrival time is generated randomly with Poisson rate.

- The burst length is generated uniformly between Lmin and Lmax (Lmin is the minimal length of bursts and Lmax the maximal one).

- The destination is one of the outputs (all the outputs have the same probability to be taken by a burst).

- The length of bursts is uniformly distributed with an average of $10 \mathrm{~ms}$. 


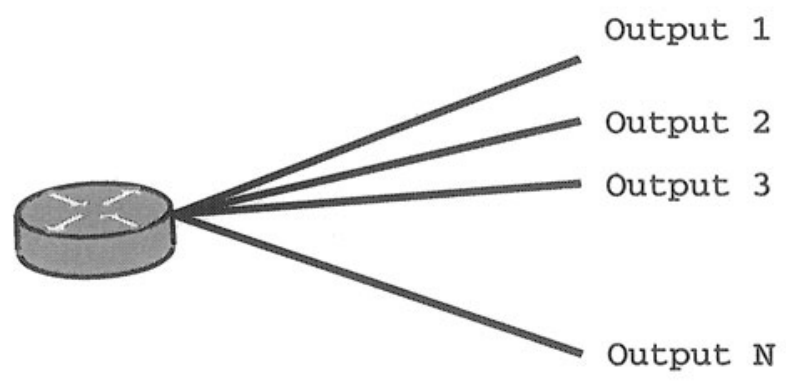

Figure 4. Simulated switch

An output may be one or more fibers. In this simulation we will consider the output with one or two fibers.

We use the same list of bursts to simulate the forwarding process according to the following switching methods:

1. Basic OBS.

2. OBS with limited buffer: in this simulation the buffer size is $0.1 *$ average size of bursts.

3. OBS with deflection: we assume that when contention occurs, all the other output are considered as an alternative route.

4. SBS: in this simulation the burst is broken into segments. All segments have the same length $(0.1 \mathrm{~ms})$, sufficient to carry several $\mathbb{P}$ packets.

The performance measure in this simulation is the blocking probability and the loss due to collision. The loss is calculated as the ratio of the dropped information and the total information sent. In case of segmented burst, we measure also the blocking probability of segments at the end of the burst, since it is the one, which carry the information with higher priority. Indeed when contention occurs, the segments, at the beginning of the second burst, are removed to forward both the first burst and the remaining part of the second burst. This parameter is particularly important to know how suitable the segmented method is, to be used with different class of service. 


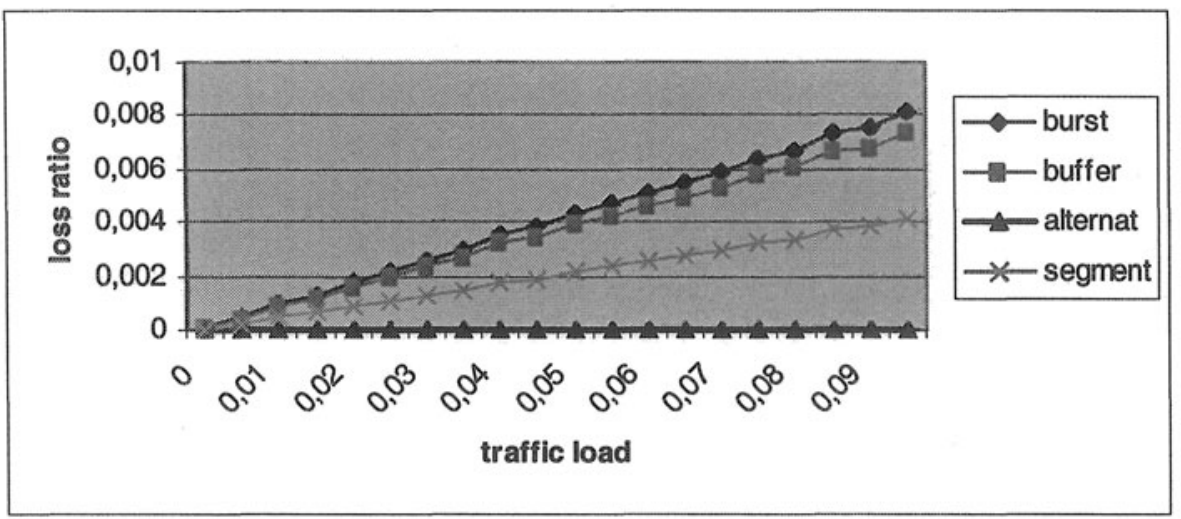

Graph 1. Switch with 10 outputs

Graph 1 shows the loss ratio of each OBS technique as function of the load. Each output contains one fiber. As expected, the blocking probability increases with the load, except for the deflection method where there is practically no loss due to the fact that the input capacity is equal to the output capacity. But in this case the number of hops is not known, thereby the delivery time will increase and eventually the burst can loop infinitely in the network. One needs some precaution to limit the lifetime of the burst such as drop the burst after a certain number of hops.

The graph also shows that segmented burst improves the performance, in term of loss ratio, by $50 \%$. This means that the number of dropped burst in the network will decrease by $50 \%$. And hence the OBS will become more stable.

Graph 2 shows that even though the number of outputs decreases, segmented burst improves the performance by $50 \%$. But for both normal OBS and segmented burst, the loss ratio have decreased due to the small number of bursts. 


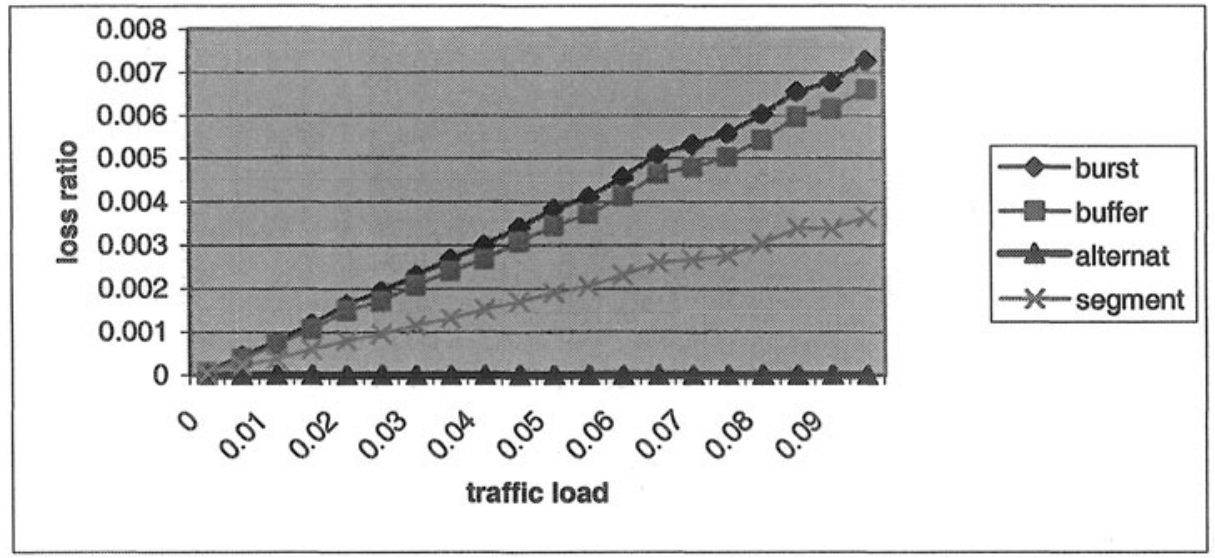

Graph 2. Switch with 5 outputs

OBS with limited buffer has the same trend than OBS, nevertheless it improves the performance with constant rate. This rate depends on the length of this buffer.

When the number of fibers in each output increases, Segmented OBS takes advantage of the added capacity. Indeed the graph 3 shows that when the number of fibers is 2 the loss is quite lower with segmented OBS when compared to the OBS loss. For example if the output number is 2 , the loss ratio falls from 0.004 to 0.002 with segmented $\mathrm{OBS}$ whereas the same loss falls only from 0.008 to 0.006 with $\mathrm{OBS}$. This result proves clearly that segmented OBS tends to exploit efficiently the available resources.

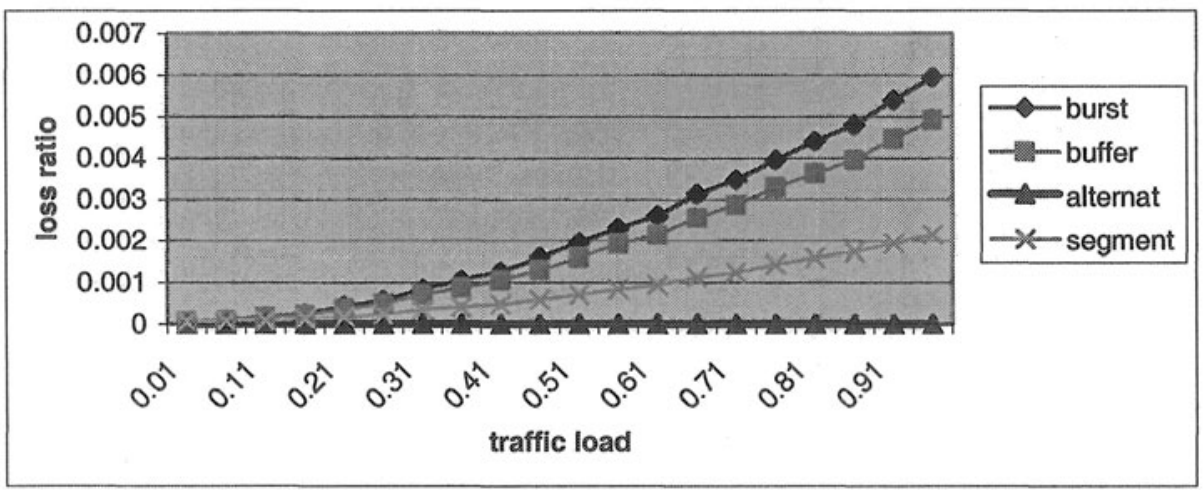

Graph 3. Switch with 10 outputs and 2 fibers in each output 
Table 2. Blocking probability of the whole burst and the last segment with low load

\begin{tabular}{lll}
\hline Load & Burst & Last segment \\
\hline 0.00 & 0.00008 & 0.00000 \\
0.01 & 0.00052 & 0.00000 \\
0.01 & 0.00090 & 0.00000 \\
0.02 & 0.00135 & 0.00000 \\
0.02 & 0.00187 & 0.00000 \\
0.03 & 0.00214 & 0.00000 \\
0.03 & 0.00260 & 0.00000 \\
0.04 & 0.00304 & 0.00000 \\
0.04 & 0.00343 & 0.00000 \\
0.05 & 0.00389 & 0.00000 \\
0.05 & 0.00426 & 0.00000 \\
0.06 & 0.00459 & 0.00001 \\
0.06 & 0.00521 & 0.00000 \\
0.07 & 0.00561 & 0.00001 \\
0.07 & 0.00588 & 0.00001 \\
0.08 & 0.00619 & 0.00001 \\
0.08 & 0.00674 & 0.00001 \\
0.09 & 0.00724 & 0.00001 \\
0.09 & 0.00753 & 0.00001 \\
0.10 & 0.00798 & 0.00001 \\
\hline
\end{tabular}

The last segment has more probability to survive contention since the dropped segments are those at the beginning of the second burst. Table 2 shows the blocking probability, for a switch with 10 outputs, for both the whole burst and the last segment of the burst. Regardless of the number of outputs, segmented burst is always showing good performance. with low traffic, the dropping probability is almost null and the last segment reaches always its destination. Indeed the loss probability is more than $7 * 10^{-3}$ whereas the last segment does not go over $1 * 10^{-5}$. This makes the segmented OBS more suitable for traffic with different class of services.

Even though the load increases, the last segment has always the biggest probability to reach destination. Table 3 shows the loss probability for the same switch with high load; the loss probability of the whole burst reaches 0.3 but the loss probability of the last segment is lower than $5^{*} 10^{-4}$. The loss probability of the last segment can be improved by decreasing the length of the segment. The Segment rank in the burst determines its priority in term of loss probability and hence the burst can carry different level of classes of services. 
Table 3. Blocking probability of the whole burst and the last segment with high load

\begin{tabular}{lll}
\hline Load & Burst & Last segment \\
\hline 0.51 & 0.03979 & 0.00004 \\
1.01 & 0.07248 & 0.00007 \\
1.51 & 0.10092 & 0.00011 \\
2.01 & 0.12596 & 0.00015 \\
2.51 & 0.14737 & 0.00016 \\
3.01 & 0.16660 & 0.00020 \\
3.51 & 0.18350 & 0.00023 \\
4.01 & 0.19936 & 0.00025 \\
4.51 & 0.21262 & 0.00027 \\
5.01 & 0.22533 & 0.00031 \\
5.51 & 0.23638 & 0.00033 \\
6.01 & 0.24752 & 0.00031 \\
6.51 & 0.25635 & 0.00035 \\
7.01 & 0.26519 & 0.00036 \\
7.51 & 0.27331 & 0.00037 \\
8.01 & 0.28059 & 0.00040 \\
8.51 & 0.28814 & 0.00039 \\
9.01 & 0.29412 & 0.00040 \\
9.51 & 0.30980 & 0.00047
\end{tabular}

\section{CONCLUSION}

DWDM has emerged as a promising technology for the next generation of networks to meet the growing bandwidth demand and fuel the exhausted fiber. OBS is one of the proposed solutions to be used with DWDM to route information in switched network. Indeed OBS has a big potential to exploit the bandwidth provided by DWDM. In this article we discussed the contention problem that occurs when two, or more bursts compete for the same output. We proposed another method to reduce the loss probability and bring more flexibility in order to use the whole capacity of the wavelength. In this method we replace the burst by several segments to avoid the loss of the whole burst in case of contention. We presented the benefits of this method and how one can use this segment to carry several classes of traffic; simulation proves that the last segment has the smallest probability to be dropped compared with the dropping probability of the burst.

In this paper, we considered only one switch with different number of outputs. Nonetheless to evaluate the edge-to-edge loss probability we need simulation with a whole network using several intermediates nodes. Another interesting issue is how to combine segmented burst with other method of 
congestion control in order to avoid completely the loss and balance the load over the network.

\section{REFERENCES}

[1] M.Yoo and C.Qio, "Optical Burst Switching [OBS]- A New Paradigm for an Optical Internet," Int'1. J. High Speed Networks, vol. 8, no.1, 1999.

[2] Jonathan Turner, "Terabit Burst Switching," Int'l. J. High Speed Networks, vol. 8, 1999.

[3] S.Verma, H.Chaskar and R.Ravikanth, "Optical Burst Switching: a viable solution for terabit IP backbone," IEEE Network December 2000.

[4] M.Yoo and C.Qiao "A New Optical Burst Switching Protocol for Supporting Quality of Service," Buffallo, New York 1998.

[5] J.Yates, M.Rumsewicz and J.LACEY, "Wavelength Converters in Dynamically" Reconfigurable WDM Networks," IEEE Communications Surveys, second quarter 1999.

[6] I. Chlamtac, A. Fumagalli, and C.J.Suh, "Multi-Buffer Delay Line Architectures for Efficient Contention Resolution in Optical Switching Nodes, IEEE Transactions on Communications", vol. 48, no. 12, pp. 2089-2098, December 2000.

[7] S.Barnes, "Migrating to an all-optical network," Telecommunications Magazine, may 2001.

[8] T.Lee, K,Lee, S.Park, "Optimal routing and wavelength assignment in WDM rings" IEEE Journal on Selected Areas in Communications, Volume: 18 Issue: 10, Oct. 2000

[9] Z.Zhang, A.S.Acampora, "A heuristic wavelength assignment algorithm for multihop WDM networks with wavelength routing and wavelength re-use" IEEE/ACM Transactions on Networking, Volume: 3 Issue: 3 , June 1995

[10] S.Yao, B.Mukherjee, S.Dixit, "Asynchronous Optical Packet-Switched Networks: a preliminary study of contention -resolution schemes" proceedings, Optical Networks Workshop January 31-February 1, 2000 Richardson, Texas

[11] D. K. Hunter, I. Andonovic "Approaches to Optical Internet Packet Switching" IEEE Communications Magazine, vol. 19, no. 9, September 2000

[12] M. Listanti, V.Eramo, R. Sabella, "Architectural and Technological Issues for Future Optical Internet Networks", IEEE Communications Volume 38, N 9 September 2000

[13] M. Yoo and C. Qiao, "A New Optical Burst Switching Protocol for Supporting Quality of Service," SPIE Proceedings, All-Optical Networking: Architecture, Control and Management Issues, Vol. 3531, Nov. 1998.

[14] C. Qiao, S.Buffalo "Labeled optical burst switching for IP-over-WDM integration" IEEE Communications Magazine, Vol. 38, No. 9, September 2000

[15] C. Qiao, M. Jeong, A. Guha, X. Zhang, J. Wei. "WDM multicasting in IP over WDM Networks," Proceedings of ICNP, Nov. 1999.

[16] M Jeong, Y Xiong, H C Cankaya, M Vandenhoute and C Qiao, "Efficient Multicast Schemes for Optical Burst-Switched WDM Networks", Int'l Conf on Communications (ICC), June 2000

[17] K. R. Venugopal, E. Ezhil Rajan and P. S Kumar "Performance Analysis of Wavelength Converters in WDM Wavelength Routed Optical Networks" Proceedings of the Fifth International Conference on High Performance Computing IEEE 1998

[18] X. Wang, H. Morikawa, T. Aoyama "Burst optical deflection routing protocol for wavelength routing WDM networks” Proc. SPIE/IEEE Opticom 2000, pp.257-266, Dallas, TX, USA, Oct. 2000. 